\title{
A Study of the Impact of Reduced Inertia in Power Systems*
}

\author{
Urmila Agrawal \\ urmila.agrawal@pnnl.gov
}

\author{
James O'Brien \\ james.obrien@pnnl.gov
}

\author{
Abhishek Somani \\ abhishek.somani@pnnl.gov
}

\author{
Thomas Mosier \\ thomas.mosier@inl.gov
}

\author{
Jeff Dagle \\ jeff.dagle@pnnl.gov
}

\begin{abstract}
Inertia in power systems plays an important role in maintaining the stability and reliability of the system by counteracting changes in frequency. However, the traditional sources of synchronous generation are being displaced by renewable resources, which often have no inherent inertia. This paper investigates the impact of reduced system inertia on several aspects of the dynamic stability of power systems, such as angular stability, primary frequency response, and oscillatory modes. This study is performed on a large-scale 2000 bus synthetic Texas model by selectively replacing synchronous generators with inverter-based generation resources. This paper also compares the analysis results obtained by the above-mentioned inertia-reduction approach of renewable integration with another approach in which the inertia constant of all synchronous generators is decreased. This paper demonstrates that only reducing the inertia of all synchronous generators in a system does not provide an accurate analysis of the challenges associated with the reduced system inertia caused by renewable integration.
\end{abstract}

\section{Introduction}

Traditionally, the majority of generators in power systems have been synchronous providing sufficient inertial frequency response during transient events. However, recent technological advancements have resulted in a significant increase in the amount of penetration of renewable energy generation resources

*This work was supported by the DOE - Office of Water Power for contract 72167A under B\&R W0102000 at the Pacific Northwest National Laboratory. Pacific Northwest National Laboratory is operated by Battelle for DOE under contract DE-AC05-76RL01830.

U. Agrawal, J. O’Brien, A. Somani and J. Dagle are with Pacific Northwest National Laboratory, Richland, WA, 99352 USA

T. Mosier is with Idaho National Laboratory, Idaho Falls, ID, 83402 USA
[1]. This is largely being driven by mandates in renewable portfolio standards which also results in the loss of large synchronous generators (mostly coal plants). These renewable energy resources are mostly connected to the grid through power-electronic devices and therefore do not contribute to the system inertia, causing a decrease in the overall system inertial response during transient events [2]. The current power system has been designed around large synchronous generators and load, with the system inertia playing an important role in determining the stability of a system. In this context, it is important to understand the impact of reduced inertia on stability of a system consisting of both synchronous generators and inertia-less generators with different level of renewable energy integration.

Several studies have been carried out to analyze the impact of increasing renewable energy penetration on the power systems. References [3] and [4] discussed the challenges associated with the high level penetration of wind generation in power systems. Reference [5] studied the effect of reduced inertia on angular stability, [6] studied the impact of increased penetration of DFIG-based wind turbine generators on transient and small-signal stability of power systems, [2] studied the impact of wind power integration on power systems frequency response. Reference [1] determined the amount of inertia required in the system during high level penetration of wind energy and [7] studied the locational impact of inertia on primary frequency response using a large scale Texas synthetic network model. NREL also performed the Eastern Renewable Generation Integration Study (ERGIS) to analyze the operational impacts of increased renewable energy penetration in the Eastern Interconnection, the detail of which can be found in [8]. So far, most of the studies are either carried out on a small scale or only applies the approach of changing inertia constant of synchronous generators to study the impact of reduced inertia.

This paper presents a thorough analysis of the impact of reduced system inertia on dynamic performance of power systems that includes angular stability, primary 
frequency response, and system oscillatory modes using a large scale synthetic network model. Two methods of reducing inertia are implemented and compared for suitability: (a) reducing the inertia constant of all synchronous generators uniformly, and (b) selectively replacing synchronous generators with non-inertial generators. Based on the results obtained, this paper demonstrates that studies carried out to analyze the impact of reduced system inertia by only changing inertia constant of synchronous generators does not accurately portray the challenges associated with the integration of renewable energy sources.

The rest of the paper is organized as follows: Section II provides some theoretical background to help understand the problem addressed in this paper along with different aspects of power systems studied in this paper to analyze the impact of reduced system inertia. Section III describes the system model and the approaches used in the paper to perform the study, Section III provides results and discussion, and section IV summarizes the conclusion.

\section{Background theory}

This section briefly describes system inertial response and it's significance in maintaining system reliable operations, and different metrics used to analyze the impact of reduced inertia on the system.

\subsection{Inertial response}

Machine inertia is the fastest possible frequency stabilization method as shown in Figure 1 and Figure 2. Inertia is innate to machines that rotate synchronously in proportion to the grid's frequency. Once a grid event occurs, the physical mass of the rotating body experiences a torque due to the change in frequency of the electrical grid. This torque induces a transfer of kinetic energy due to the machine's rotation to the electrical grid. The transfer of energy from the machine to the electrical grid works to slow the rate of change of frequency (ROCOF) of the electrical grid instantaneously. Per unit inertia constant $\mathrm{H}$ is defined as kinetic energy in watt-seconds at rated speed, $\omega_{0}$, divided by the apparent power in per unit base $P_{m v a}$,

$$
H=\frac{J \omega_{0}^{2}}{2 P_{m v a}},
$$

where $J$ is the rotor moment of inertia in $k g-m^{2}$. This inertia constant depends on the rotor moment of inertia, $J$, and this moment of inertia is equal to the weight of the rotating parts multiplied by the square of radians of gyration [9].

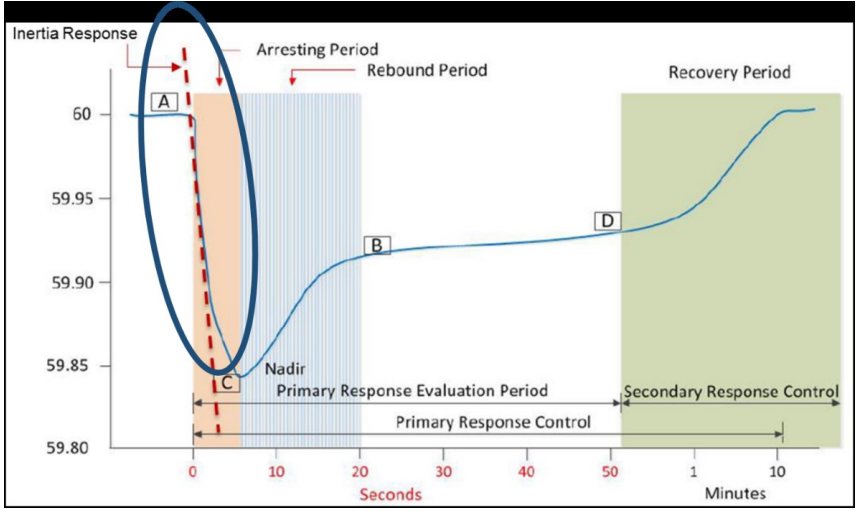

Figure 1. Archetypal frequency pattern after frequency deviation event. Reproduced from [10]

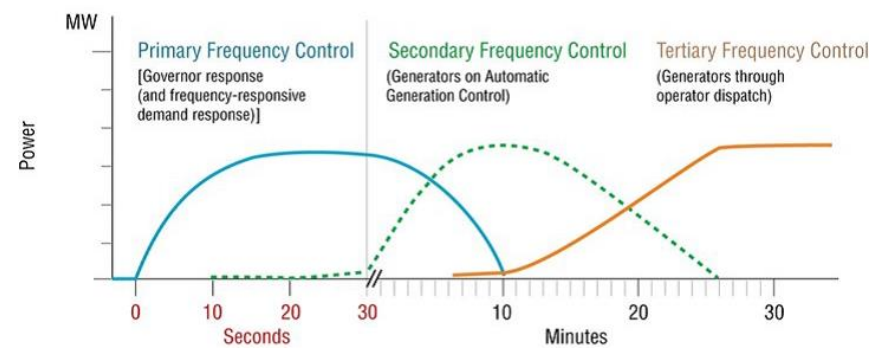

Figure 2. Sequence of frequency response and recovery actions. Reproduced from [11].

System inertia plays a great role in ensuring reliability of grid operations by arresting the initial change in frequency after an event. With increasing renewable integration, this inertial response, provided by rotating machines, has been continuously decreasing and thereby affecting the system primary frequency response and therefore system reliable operations. In addition to the primary frequency response, reduction in system inertia also affects transient angle stability, as discussed later in greater detail, affecting system's stability margin.

\subsection{Rate of Change of Frequency (ROCOF)}

ROCOF gives the measure of the change in frequency with time $(\mathrm{df} / \mathrm{dt})$ following a system event, and is determined by the system inertia and the magnitude of the system event [12]. Traditionally, ROCOF was less significant for system stability studies as power systems mainly consisted of synchronous generators limiting the ROCOF. However, with increasing renewable integration, ROCOF has become relevant for system dynamic stability studies. Reduced system inertia can result in large ROCOF values, which in turn can affect system reliable operations because 
of mechanical limitations of individual synchronous machines as described in [12].

\subsection{Transient angle stability}

"It is the ability of the system to remain in synchronism when subjected to large disturbances [13]." For the system to maintain stability following a large transient events, the rotor angular separation needs to be within a certain limit as determined by equal area criterion method.

\subsection{Frequency nadir}

The frequency nadir is given by the minimum frequency at which the system frequency is arrested following a transient event, such as loss of a generator. This nadir point is determined by the spinning reserve available for primary frequency response and system inertia. The frequency nadir determines if the under-frequency load-shedding is initiated or not such that if the frequency decreases below certain specified value then load-tripping relays will be activated.

\subsection{Small-signal stability}

"It is the ability of the system to maintain synchronism when subjected to small disturbances [13]". The small-signal stability margin of a system is determined by system modes, which are given by the eigenvalues of system state matrix,

$$
\lambda_{i}=\sigma_{i}+j \omega_{i}
$$

where $j=\sqrt{-1}$ and $\lambda_{i}$ is the $i^{t h}$ mode of the system. These modes are characterized by their frequencies given by

$$
f_{i}=\frac{\omega_{i}}{2 \pi} \quad \mathrm{Hz}
$$

and damping coefficients given by $\sigma_{i}$. The damping ratio, $\zeta_{i}$, of a system mode is given by

$$
\zeta=\frac{-\sigma_{i}}{\sqrt{\sigma_{1}^{2}+\omega_{1}^{2}}}
$$

The damping ratio of system modes give a measure of the small signal stability margin of the system. A system consisting of a mode with damping ratio close to zero indicates that the system is vulnerable to system events. Therefore, it is very important that system modes have enough damping ratio $(>5 \%)$ such that the system still exhibits positive damping ratio after occurrence of a transient event. Usually, power system stabilizers (PSS) are used to improve the damping ratio of system modes. These PSS parameter settings are system specific and are tuned to improve the damping ratio of these system modes.

Past studies, which are based on reducing system inertia by reducing the inertia of synchronous generators, have concluded that reducing the system inertia results in an increase in the frequency of system modes resulting in faster frequency dynamics in the system $[7,14]$.

\section{System modeling and simulation scenarios}

This paper uses a 2000-bus Texas synthetic network model to carry out the simulations, the details of which can be found in [15]. All the simulations were carried out using the PowerWorld Simulator. In the Texas base-case model, the total load was close to 67,000 MW with renewable energy penetration, mostly wind, composing $13.37 \%$ of the load served. The frequency response reserve capacity was equal to $13,500 \mathrm{MW}$. The total system inertia was equal to $381.34 \mathrm{GW} \cdot \mathrm{s}$, which was obtained by taking sum of the inertia of all synchronous generators in the system model. The model consists of eight areas: Coast, East, North, North Central, South, South Central, West, and Far West. The wind and solar generators were modeled using type-4 machine model equipped with an exciter and connected to the grid through power-electronics devices. The type-4 machine model was used primarily because modern wind generators are mostly power-electronics based with no inherent inertia. The parameters used for modeling wind and solar generators were taken from the existing WECC (Western Electricity Coordinating Council) system model for 2025 heavy summer operating case.

The two approaches used to study the impact of reduced system inertia are:

\subsection{Reducing inertia of synchronous generators (Approach-1)}

In this approach, the inertia of synchronous generators were reduced to study the impact of the reduced inertia without making any changes to the renewable integration level. For this, the inertia constant, as defined in (1), in the generator machine model was reduced by a given percentage for all the synchronous generators in the system. All other parameters in the machine model and exciter model were unchanged. 


\subsection{Replacing synchronous generators with inertia-less generators (Approach-2)}

Unlike approach-1, this is a realistic approach to study the challenges associated with the increased penetration of inertia-less generators connected to the grid through power-electronics. In this approach, the conventional synchronous generators were replaced with type-4 inertia-less wind generators equipped with an exciter. The synchronous generators to be replaced were selected across all areas, such that the proportion of inertia among different areas were similar to that in the base-case, to obtain a wind penetration level of $20 \%, 30 \%, 40 \%, 55 \%, 60 \%, 65 \%$ and $70 \%$. Figure 3 shows the percentage change in the inertia of the system corresponding to the different level of wind penetration. This percent change in the system inertia was then used in the first approach to modify the inertia of synchronous generators such that the same set of values of the total system inertia were used in the two approaches. Also, the dispatch, system load and stress were same for all the cases in both the approaches.

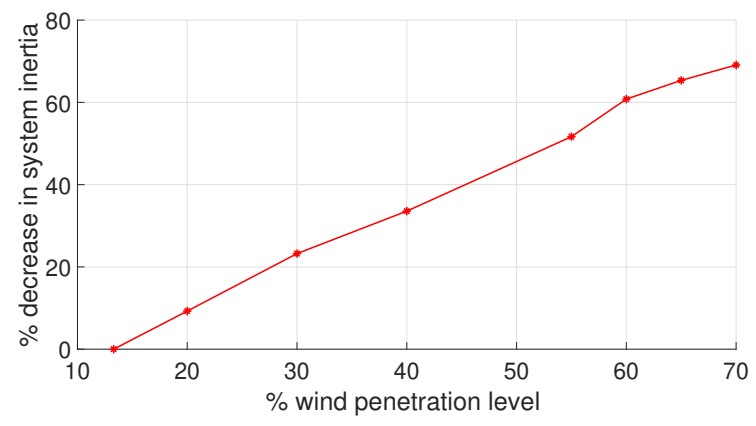

Figure 3. \% decrease in system inertia because of increased wind penetration level

\section{Results and Discussions}

During transient events, inertia provided by synchronous generators counteracts the changes in frequency, and therefore helps with maintaining angular and frequency stability. This paper studies the impact of reduced system inertia on these aspects of power systems transient stability, which includes rotor angle deviations and primary frequency response. This paper also analyzes the impact of reduced system inertia on the system small-signal stability margin. For this, system modes were estimated for the two inertia-reduction approaches.

Transient-stability analysis was performed to obtain maximum rotor angle, minimum rate of change of frequency (ROCOF) and frequency nadir following a

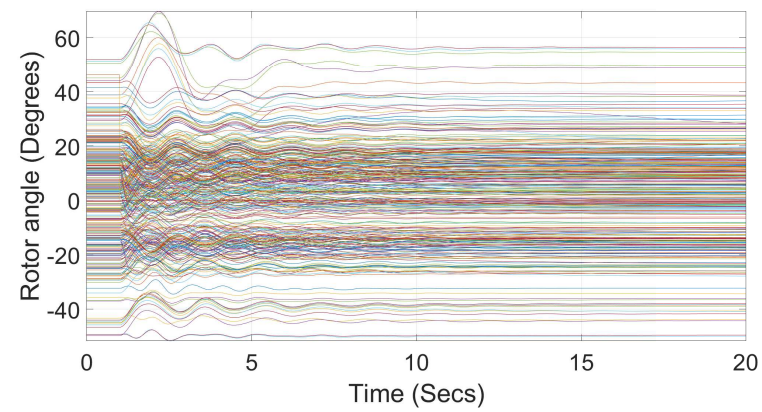

(a) Rotor angle

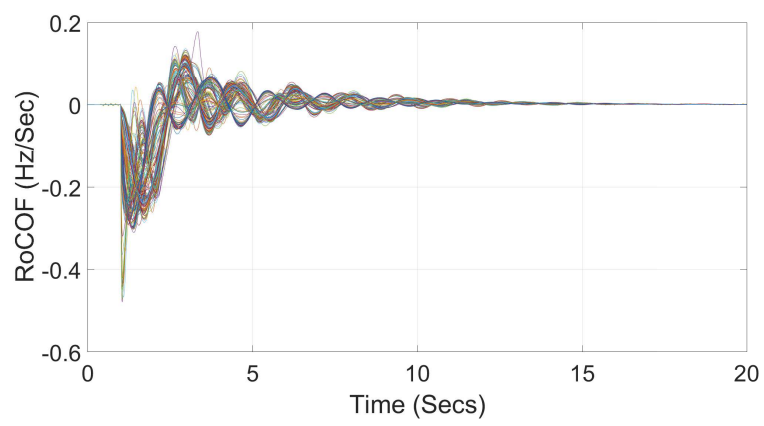

(b) Rate of change of frequency

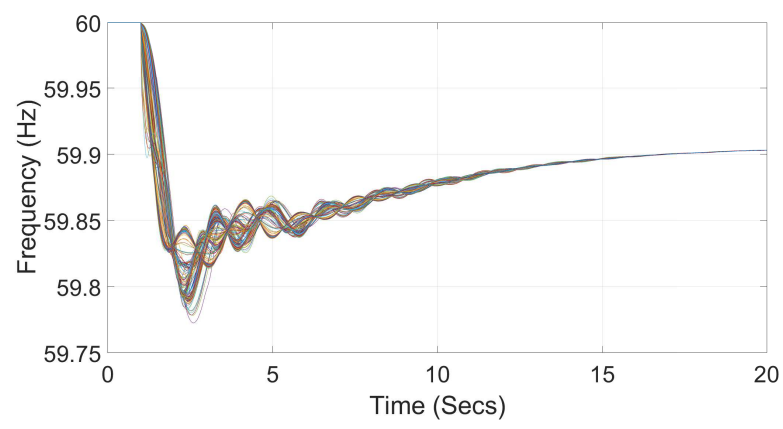

(c) Bus frequency

Figure 4. Transient stability analysis results obtained for the base-case.

transient event. Modal analysis was performed to obtain the estimates of system modes using ringdown oscillations caused by the transient event. The transient event comprised of tripping of two $1350 \mathrm{MW}$ nuclear generators located in the coast area. Following the trips, maximum rotor angle, frequency nadir and minimum ROCOF values were obtained. The maximum rotor angle is given by the maximum of the rotor angle deviation observed in any generator in the system following the transient event; frequency nadir is given by the minimum frequency observed in any of the buses in the system; and minimum ROCOF is given by the minimum of the ROCOF observed in any of the system buses. Figure 4 show the generator rotor angle, ROCOF, 


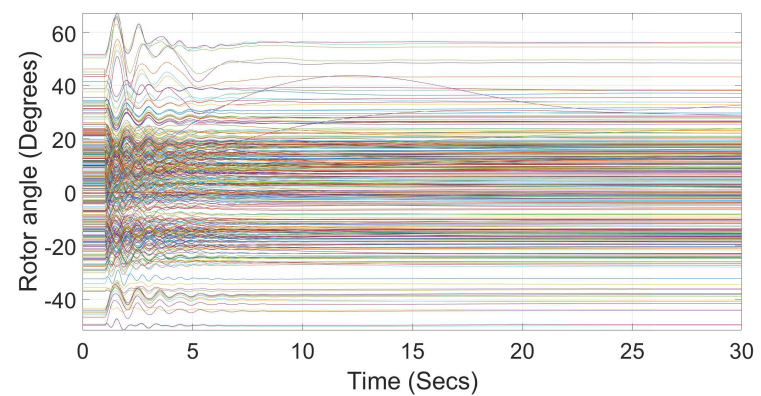

(a) Rotor angle

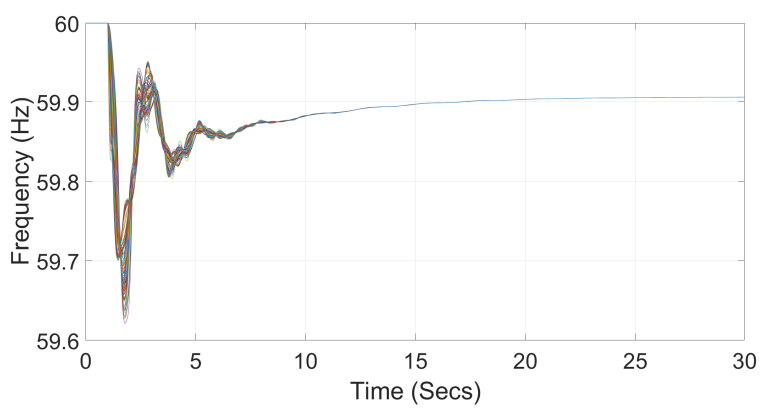

(b) Bus frequency

Figure 5. Transient stability analysis results obtained for the approach-1 with inertia reduced by $70 \%$ as compared to the base-case.

and bus-frequency observed in the system following the transient event for the base case with a wind penetration level of $13.37 \%$ and total system inertia of $381.34 \mathrm{GW} \cdot \mathrm{s}$; Figure 5 and Figure 6 are associated with the case having system inertia reduced by close to $70 \%$ using approach-1 and approach-2, respectively. The rindown oscillation caused by this transient event, as shown in Figure 4(c) for the base-case, was then used to obtain system mode estimates using Prony analysis, described in [16], to estimate frequency of system modes. Figure 7 shows generators in different areas oscillating against each other, following the transient event, with the generators located in the same colored area oscillating together.

For modal analysis, rotor angle measurements of synchronous generators were used to calculate frequency measurements, given by the time derivative of rotor angle measurements. The estimated frequency measurements measured at different generator buses were then used for performing modal analysis. The simulated data were generated at the rate of 120 samples per second and later decimated to a frequency of 8 samples per second. Usually, for modal analysis, the data are decimated to a frequency of 4-5 samples per second as the system modes of interest are in the range

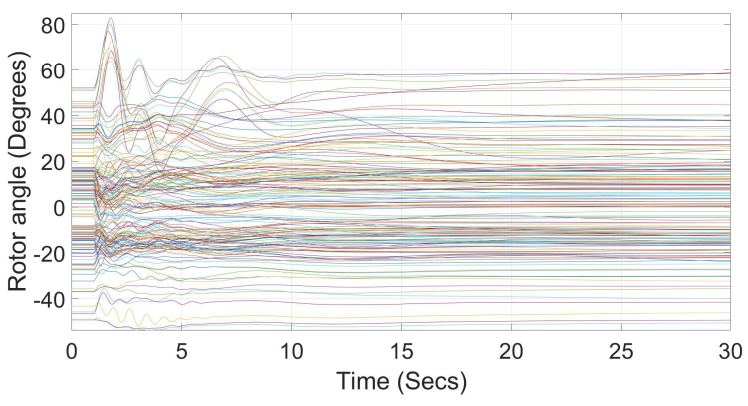

(a) Rotor angle

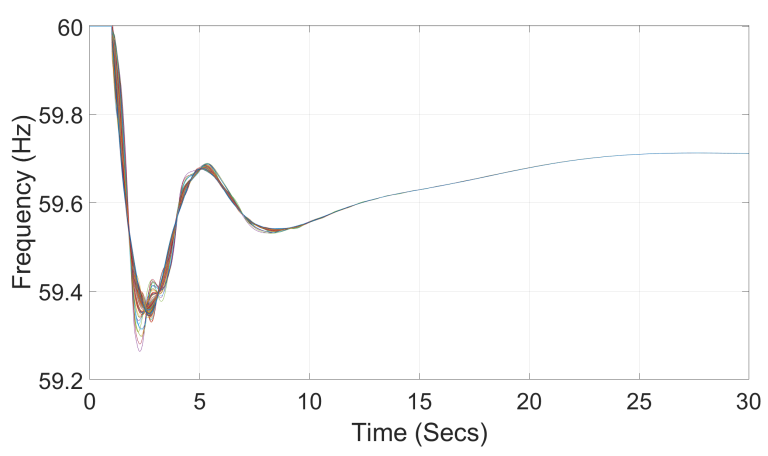

(b) Bus frequency

Figure 6. Transient stability analysis results obtained for the approach-2 with inertia reduced by $70 \%$ as compared to the base-case.

of up to $2 \mathrm{~Hz}$ [17]. However, with reduced system inertia, to account for the possibility of existence of faster frequency dynamics, as described in [7] and [14], a higher sampling frequency was used. The Prony analysis based on multiple-signals, described in [16], was used to obtain system mode estimates.

Figure 8 through Figure 10 summarizes the results obtained using the two inertia-reduction approaches. In the plots, the results obtained for a $0 \%$ decrease in the system inertia corresponds to the base-case. Figure 8 illustrates the impact of system inertia on the primary frequency response. Figure 8(a) compares the amount of frequency response reserve (FRR) available for different cases in each simulation scenario. For the first approach in which only the inertia constant of the generator machine model was changed thus the FRR is retained. However, in the second approach, synchronous online generation is displaced by wind. Wind generation is not assumed to have any FRR available, and hence, did not contribute to the primary frequency response. Due to this, the amount of total FRR decreases with this approach. This availability of the primary frequency response affects the frequency nadir and, as expected, the frequency nadir has lower 


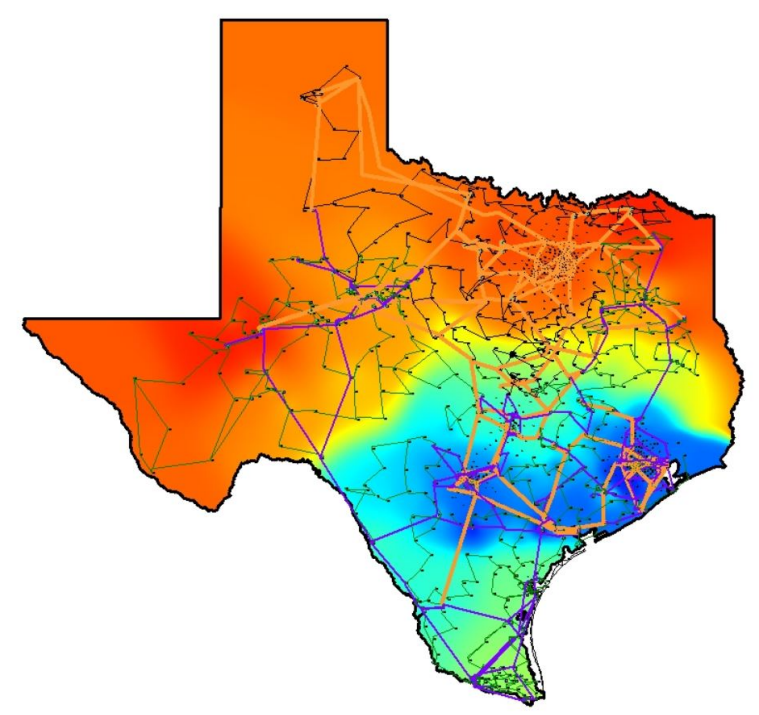

Figure 7. Illustration of generators of different areas oscillating against each other.

value for the second approach as compared to the first approach as shown in Figure 8(c). The decrease in the FRR was dependent on the generators that were replaced and so the decrease in the FRR was not consistent for different wind penetration level as seen in Figure 8(a). The ROCOF for the two approaches closely matched, illustrating that this metric is highly dependent on the total system inertia for transient events with similar impact.

Figure 9 compares the effect of the reduced system inertia on the angular stability of the system. During transient events, if the rotor angle increases beyond some maximum value, then the generator can go out of synchronism. As can be seen in Figure 9, the maximum rotor angle observed following the transient event for the two approaches follow different pattern even though the system inertia for both the approaches were equivalent in the different cases. In the first approach, the observed maximum rotor angle decreased, while in the second approach, it increased. This result is quite expected. As the synchronous generators are replaced with the wind generators, each of the remaining fewer synchronous generators has to contribute more power for the same amount of lost generation and thus results in an increased rotor angle. For the first approach, a small decrease in the maximum rotor angle deviation with decrease in the system inertia might have been caused by the redistribution of the power contribution of different generators, during the transient event, caused by the decrease in the kinetic energy (inertia) of all synchronous generators. Therefore, based on the result for the second approach, it can be said

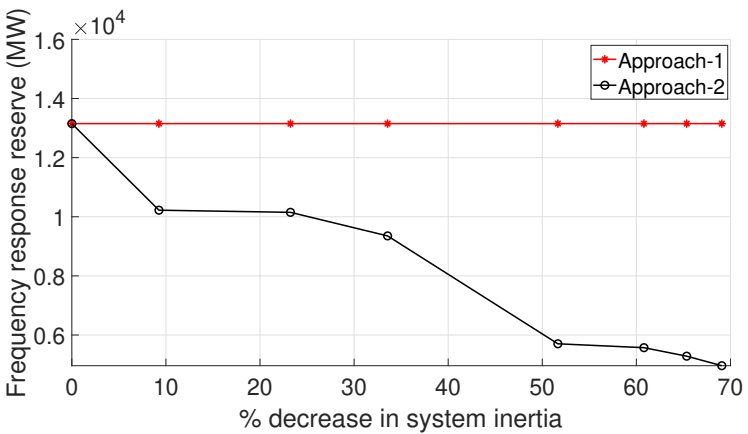

(a) Frequency response reserve (MW)

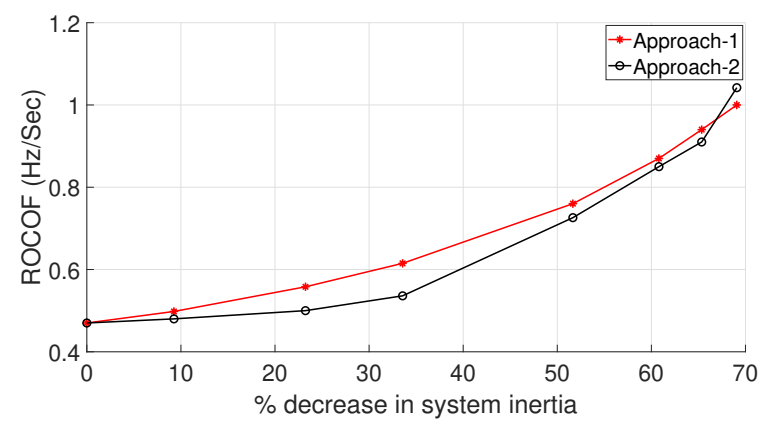

(b) Rate of change of frequency

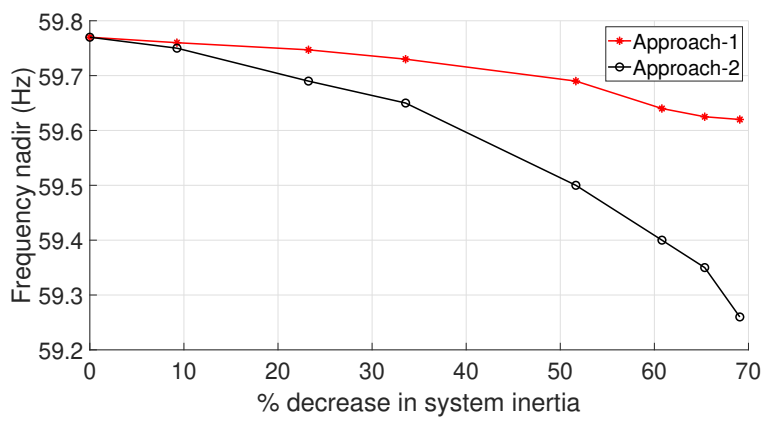

(c) Frequency nadir

Figure 8. Comparison of the available primary frequency response for the two inertia-reduction approaches.

that increasing penetration of inertia-less generators reduces the angular-stability margin of the system and can potentially lead to a first-swing instability issue at some point. This was not observed in the results obtained using the first approach.

As synchronous generators are replaced with non-inertial generators, fewer generators are available to participate in oscillations. Thus, change in frequency and damping ratio of system modes is expected based on the dynamics of the online synchronous generators and participation of these generators in different oscillatory modes. Figure 10 and Figure 11 compare the effect of 


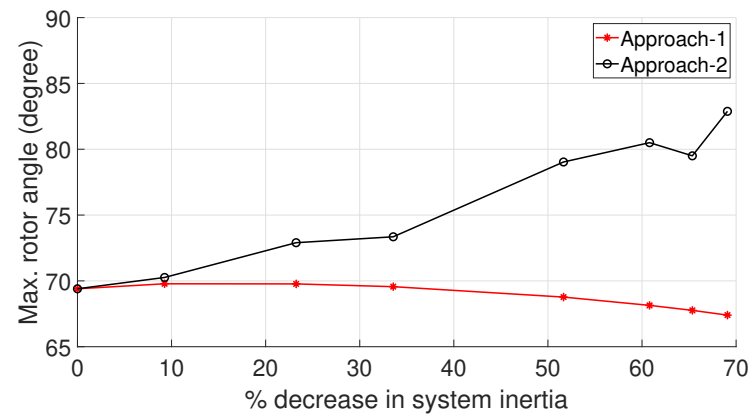

Figure 9. Comparison of the effect of reduced system inertia on the maximum rotor angle observed, following a transient event, for the two approaches.

reduced system inertia on the frequency and damping ratio of three system modes. For approach-2, where synchronous generators are replaced with non-inertial generators, some changes in system modes were observed. However, the change in the frequency of system modes did not follow any trend with the change in system inertia. Similar results were observed for damping ratio of system modes, as can be seen in Figure 11. Therefore, based on the results obtained, it can be said that with increased renewable integration, any changes in system modes will depend on the dynamics of remaining online synchronous generators and not so much on system inertia. Therefore, damping ratio of system modes should not be a concern when considering renewable integration. However, when the reduction in system inertia was achieved by decreasing inertia of generators, the impact of system inertia was clearly observed in system modes. As can be seen in Figure 10, the system mode frequency increased consistently with the decrease in the inertia of the generators. This result was quite expected as the machines with lower mass (inertia) can oscillate at higher frequency as compared to the ones with higher mass. Therefore, simply reducing inertia of machines across the system would result in higher frequency system dynamics, which is not the case when synchronous generators are replaced by inverter-based generators.

\section{Conclusion}

This paper analyzes the impact of reduced inertia in power systems using a large scale synthetic Texas model. The analysis is carried out by selectively replacing synchronous generators with inertia-less wind plants. As illustrated in the paper, the increasing penetration level of inertia-less generators affects the angular stability margin and primary frequency response of the system. As the proportion of inertia-less

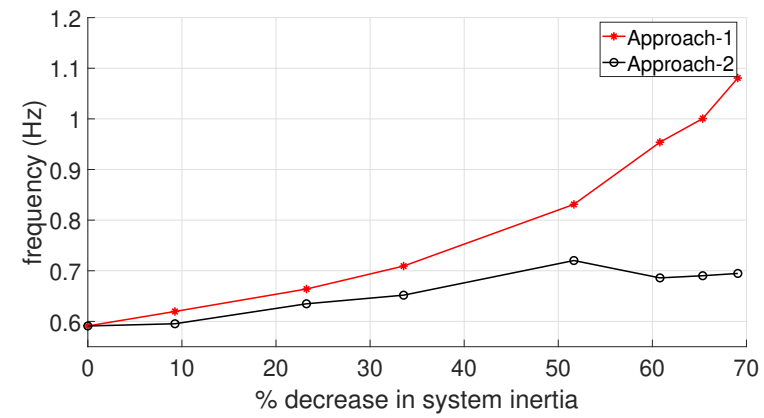

(a) Mode-1

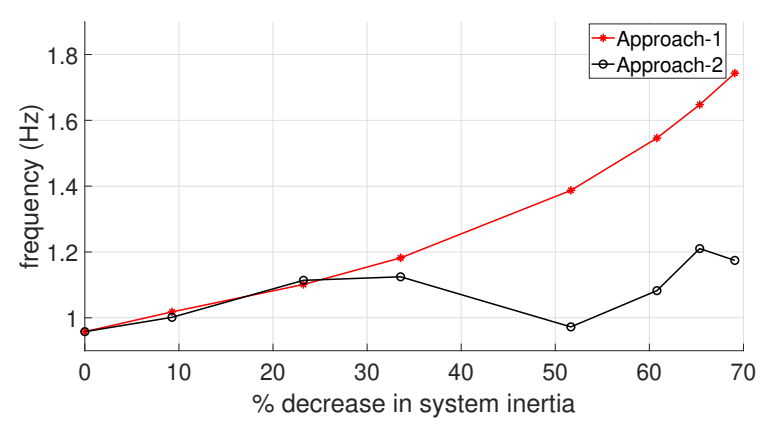

(b) Mode-2

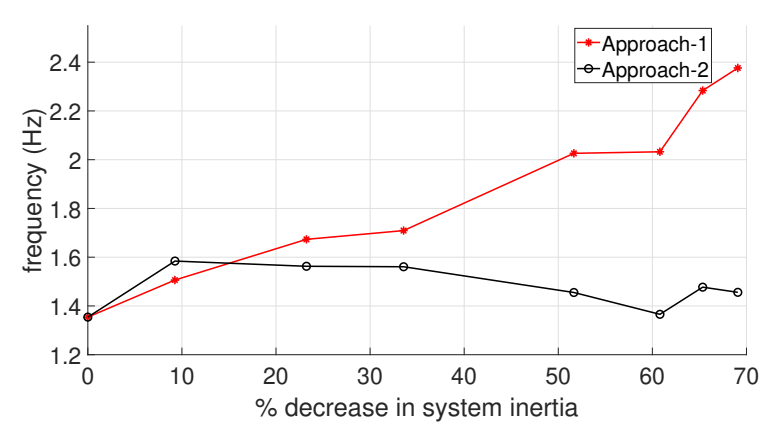

(c) Mode-3

Figure 10. Comparison of the impact of reduced system inertia on frequency of system modes for the two inertia-reduction approaches.

generators increased, the rotor angle deviation observed, following a transient event, also increased indicating a lower angular stability margin. Similarly, the available spinning reserve decreased as fewer generators were available to contribute to the primary frequency response. This calls for a need of the fast frequency response (FFR) generators, which can also include synthetic inertia and battery storage, that can quickly contribute to the system frequency recovery following a generation-loss. The impact of reduced inertia on the system modes was not observed and changes in these modes were more likely caused by the change in system topology with fewer generators available to participate in system oscillations. This paper also demonstrated that 


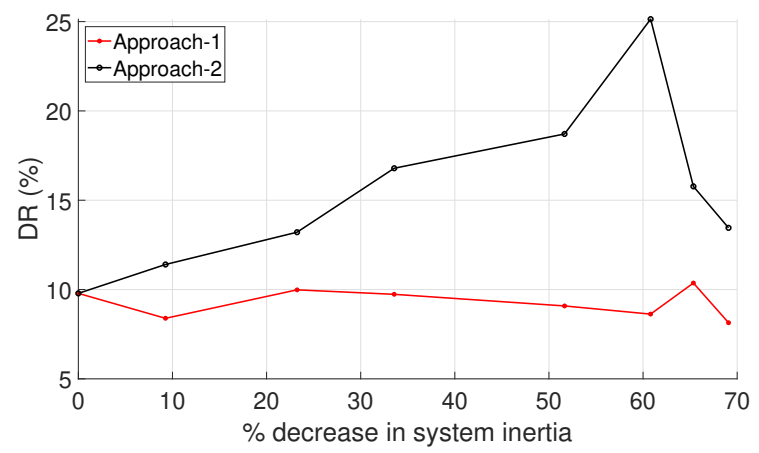

(a) Mode-1

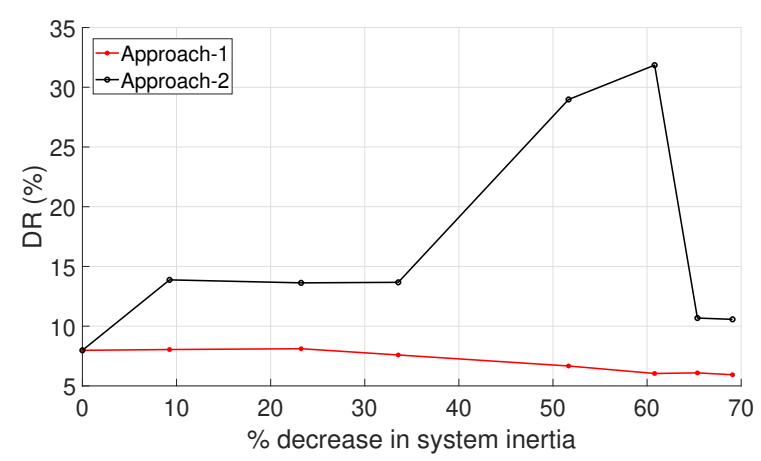

(b) Mode-2

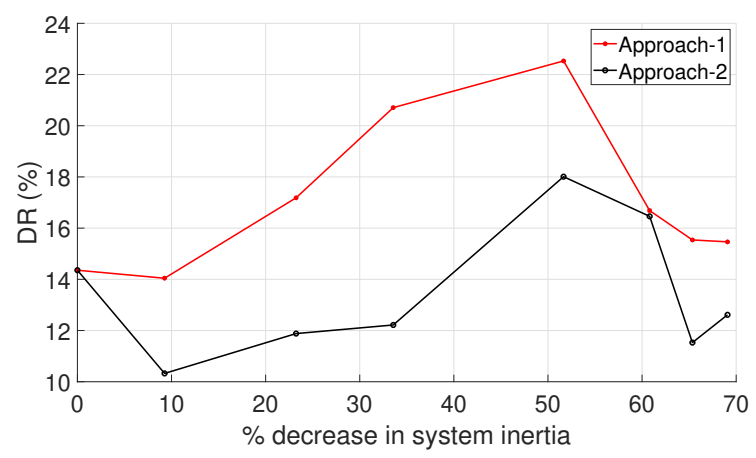

(c) Mode-3

Figure 11. Comparison of the impact of reduced system inertia on the damping ratio of system modes for the two inertia-reduction approaches.

only reducing inertia of synchronous generators does not provide an accurate analysis of the challenges associated with the increasing level of renewable integration. As a part of future work, the capability of wind and solar plants to provide fast frequency response will be explored as measures to mitigate the impacts of higher level of renewable energy integration in the grid.

\section{References}

[1] N. Farrokhseresht, H. C. Oréstica, and M. R. Hesamzadeh, "Determination of acceptable inertia limit for ensuring adequacy under high levels of wind integration," in 11th International Conference on the European Energy Market (EEM14), pp. 1-5, May 2014.

[2] H. R. Chamorro, M. Ghandhari, and R. Eriksson, "Wind power impact on power system frequency response," in 2013 North American Power Symposium (NAPS), pp. 1-6, Sept 2013.

[3] G. Lalor, A. Mullane, and M. O’Malley, "Frequency control and wind turbine technologies," IEEE Transactions on Power Systems, vol. 20, pp. 1905-1913, Nov 2005.

[4] J. Conto, "Grid challenges on high penetration levels of wind power," in 2012 IEEE Power and Energy Society General Meeting, pp. 1-3, July 2012.

[5] X. Chen, W. Du, and H. F. Wang, "Power system angular stability as affected by the reduced inertia due to wind displacing synchronous generators," in 2017 2nd International Conference on Power and Renewable Energy (ICPRE), pp. 402-406, Sept 2017.

[6] D. Gautam, V. Vittal, and T. Harbour, "Impact of Increased Penetration of DFIG-Based Wind Turbine Generators on Transient and Small Signal Stability of Power Systems," IEEE Transactions on Power Systems, vol. 24, pp. 1426-1434, Aug 2009.

[7] T. Xu, W. Jang, and T. J. Overbye, "Investigation of inertia's locational impacts on primary frequency, response using large-scale synthetic network models," in 2017 IEEE Power and Energy Conference at Illinois (PECI), pp. 1-7, Feb 2017.

[8] "https://www.nrel.gov/grid/ergis.html."

[9] P. Tielens and D. Van Hertem, "The relevance of inertia in power systems," Renewable and Sustainable Energy Reviews, vol. 55, pp. 999-1009, 2016.

[10] "Essential reliability services whitepaper on sufficiency guidelines," tech. rep., North American Electric Reliability Corporation, 2016.

[11] J. H. Eto, J. Undrill, C. Roberts, P. Mackin, and J. Ellis, "Frequency control requirements for reliable interconnection frequency response," 2018.

[12] "Rate of Change of Frequency (ROCOF) withstand capability ENTSO-E guidance document for national implementation for network codes on grid connection," tech. rep., ENTSOE (European Network of Transmission System Operators for Electricity, 2017.

[13] P. Kundur, "“power system stability and control"," Electric Power Research Institute, Power System Engineering Series, McGraw-Hill Inc, 1994.

[14] A. Ulbig, T. S. Borsche, and G. Andersson, "Impact of low rotational inertia on power system stability and operation," IFAC Proceedings Volumes, vol. 47, no. 3, pp. 7290-7297, 2014.

[15] https://icseg.iti.illinois.edu/synthetic-power-cases/texas 2000 june2016/, "Texas 2000-june 2016.".

[16] D. J. Trudnowski, J. M. Johnson, and J. F. Hauer, "Making prony analysis more accurate using multiple signals," IEEE Transactions on Power Systems, vol. 14, pp. 226-231, Feb 1999.

[17] J. W. Pierre, D. J. Trudnowski, and M. K. Donnelly, "Initial results in electromechanical mode identification from ambient data," IEEE Transactions on Power Systems, vol. 12, pp. 1245-1251, Aug 1997. 